Research Paper

\title{
Polydatin, Natural Precursor of Resveratrol, Promotes Osteogenic Differentiation of Mesenchymal Stem Cells
}

\author{
Adriana Di Benedetto ${ }^{\circledR}$, Francesca Posa ${ }^{1,2}$, Salvatore De Maria3 ${ }^{3}$, Giampietro Ravagnan ${ }^{3}$, Andrea Ballini ${ }^{4}$,

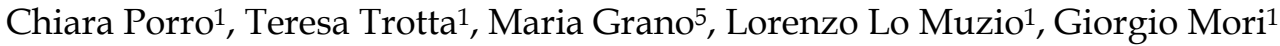 \\ 1. Department of Clinical and Experimental Medicine, University of Foggia, Foggia, Italy \\ 2. Max Planck Institute for Medical Research and Institute of Physical Chemistry, Department of Biophysical Chemistry, University of Heidelberg, Germany \\ 3. Glures srl. Unità Operativa di Napoli, spin off accademico dell'Università di Venezia Cà Foscari, Italy \\ 4. Department of Basic Medical Sciences, Neurosciences and Organs of Senses, University of Bari, Bari, Italy \\ 5. Department of Emergency and Organ Transplantation, Section of Human Anatomy and Histology, University of Bari, Bari, Italy \\ A.D. and F.P. equally contributed to this work. \\ \ Corresponding author: Adriana Di Benedetto. Email: adriana.dibenedetto@unifg.it \\ (c) Ivyspring International Publisher. This is an open access article distributed under the terms of the Creative Commons Attribution (CC BY-NC) license \\ (https://creativecommons.org/licenses/by-nc/4.0/). See http://ivyspring.com/terms for full terms and conditions.
}

Received: 2017.11.30; Accepted: 2018.04.09; Published: 2018.06.13

\begin{abstract}
Bone loss and fractures are consequences of aging, diseases or traumas. Furthermore the increased number of aged people, due to the rise of life expectancy, needs more strategies to limit the bone loss and regenerate the lost tissue, ameliorating the life quality of patients. A great interest for non-pharmacological therapies based on natural compounds is emerging and focusing on the oligostilbene Polydatin, present in many kinds of fruits and vegetables, when resveratrol particularly in red wines. These molecules have been extensively studied due to their antioxidant and anti-inflammatory effects, showing more recently Resveratrol the ability to enhance osteogenic differentiation and bone formation. However, the clinical applications of Resveratrol are limited due to its low bioavailability and rapid metabolism, while its natural glycosilated precursor Polydatin shows better metabolic stability and major abundance in fresh fruits and vegetables. Nevertheless the role of Polydatin on osteogenic differentiation is still unexplored. Mesenchymal stem cells (MSCs) from dental tissues, such as dental bud stem cells (DBSCs), are able to differentiate toward osteogenic lineage: thus we investigated how Resveratrol and Polydatin influence the differentiation of DBSCs, eventually affecting bone formation. Our results showed that Polydatin increases MSCs osteogenic differentiation sharing similar properties with Resveratrol. These results encourage to deepen the effects of this molecule on bone health and its associated mechanisms of action, wishing for the future a successful use in bone loss prevention and therapy.
\end{abstract}

Key words: Mesenchymal Stem Cells (MSCs), phytochemicals, Polydatin, Resveratrol, osteogenic differentiation, bone health.

\section{Introduction}

Aging induces in humans a natural and gradual loss of bone mass resulting in skeletal complications. The rise of life expectancy in most developed countries results in an increased number of osteoporotic patients with high risk of fractures. Nevertheless, numerous other diseases, with different etiopathogenesis, lead to complications such as necrosis and degeneration of bone tissue. To improve the life quality of patients with osteoporosis or damaged bone tissue, either preventive countermeasures, addressed to limit the bone loss, or tissue regeneration strategies targeted to rebuilt the lost bone are needed. The pharmacological therapies often present advantages or side effects depending on the patients to which are administered, thus generating a great interest in the community for non-pharmacological strategies (1), such as food supplements containing natural elements and compounds. For instance, several studies conducted on humans at different ages reported that high fruit 
intake correlates with higher bone mineral density (BMD) (2), lower hip fracture incidence (3) and better healing after periodontal therapy, preventing tooth loss (4). The antioxidant properties of phytochemicals present in fruits may be liable for such positive effects on bone health. Resveratrol (Res) is a polyphenolic oligostilbene present in many kinds of fruits and plants such as Polygonum Cuspidatum, grapes, cranberries, nuts and other plants. The evidences of its multiple biological effects including cardioprotective, anticancer, neuroprotective, antioxidant and anti-inflammatory effects (5-8) suggested that Res could be an efficacious food supplement for age-related degenerative diseases such as osteoporosis. Indeed in vitro studies reported that Res directly acts on bone-forming osteoblast cells (9-12) and their precursors (13-16) stimulating their differentiation. Accordingly, evidences from in vivo studies conducted on different animal models, encourage human trials to test the therapeutic efficacy of Res supplementation on bone turnover $(17,18)$. Actually, low bioavailability and clearance of Res could limit the translation of concentrations, experimentally established, to clinical doses efficacious to achieve beneficial health effects $(19,20)$. By contrast the natural precursor of Res, the glucoside Polydatin (Pol), present in fruits and plants, showed better oral absorption and metabolic stability than Res, since its serum concentration was 3-4 times higher after oral administration at the same dosage (21), with the further advantage of being more abundant than Res (22-24). Pol shares with Res high antioxidant properties (25) showing even superior ability to modulate oxidative stress (21). Despite a role in ameliorating bone health has been established for Res, there are not studies exploring the effect of its precursor Pol on osteoblast differentiation. Interestingly, very recent studies evidenced that Pol improved bone marrow-derived mesenchymal stem cells (BMSCs) survival, protecting them against oxidative injury (26), in spite of inhibiting proliferation and inducing apoptosis via $\beta$-catenin signaling at high dose in human osteosarcoma cells (27). Taken together this data encouraged to test and compare the effects of Pol and Res on osteoblast differentiation from mesenchymal stem cells. We and other authors have previously demonstrated that the dental tissues are good sources of cells with excellent mesenchymal stem features that represent an optimal model of cell differentiation toward osteogenic lineage (28-38). In the present study we have investigated in vitro how administration of Res and Pol could influence the osteogenic differentiation of Dental Bud Stem Cells (DBSCs). Our results showed that Res improves the osteogenic capacity of DBSCs and demonstrated for the first time that also its precursor Pol can enhance osteogenic differentiation of these cells. This finding encourages to further investigate the effects of Pol on bone and opens new perspectives for its use as a therapeutic agent in the light of higher absorption and abundance of Pol versus Res.

\section{Material and Methods}

Antibodies were from Abnova. Ascorbic acid, $\beta$-glicerophosphate, Alizarin red powder, alkaline phosphatase staining kit, MTT, were from Sigma Aldrich. Resveratrol and Polydatin extracted from Polygonum Cuspidatum, according to the procedure described in the Patent EP1292320B1, were provided by Prof. Ravagnan. Resveratrol and polydatin were dissolved in ethanol at $100 \mathrm{mM}$ stock solutions (39). Intermediate $1 \mathrm{mM}$ and $0.1 \mathrm{mM}$ stock solutions were freshly prepared by diluting in ethanol, $100 \mathrm{mM}$ stocks, 1:100 and 1:1000 respectively. Intermediate $1 \mathrm{mM}$ and $0.1 \mathrm{mM}$ stock solutions were diluted 1:1000 in culture medium just prior the use, to reach the final concentrations of $1.0 \mu \mathrm{M}$ and $0.1 \mu \mathrm{M}$. CTR samples carried 1:1000 dilution of vehicle. Control without vehicle didn't show any difference compared to control with vehicle. For this reason, the control without vehicle was not included in the data for simpleness.

\section{Patients and Dental Bud Stem Cells Cultures}

Third molars tooth buds were obtained from 15 healthy pediatric male donors (8-12 years) underwent to extractions for orthodontics reasons. Parents of each patient gave informed consent to tooth extraction with piezo-surgery technology. The study was approved by the Institutional Review Board of the Department of Clinical and Experimental Medicine, University of Foggia. The central part of the DBs, was dissected in small pieces and digested in presence of $3 \mathrm{mg} / \mathrm{mL}$ collagenase I, $4 \mathrm{mg} / \mathrm{mL}$ dispase (Gibco Ltd., Uxbridge, UK) for 1 hour at $37^{\circ} \mathrm{C}$ by shacking. Suspension was filtered and the cells were expanded, characterized for mesenchymal stem markers expression and differentiated toward osteogenic lineage as previously described $(28,40)$.

\section{Western blot}

Detection of protein levels was performed by western blot. DBSCs lysates were cleared by centrifugation at $13000 \mathrm{rpm}$ for $15^{\prime}$ at $4^{\circ} \mathrm{C}$. The protein concentration of the recovered supernatant was established by protein assay (BIORAD). Same amounts of proteins for each sample were separated by SDS-PAGE, transferred to nitrocellulose membranes (Amersham, UK) using the Trans-Blot 
(Biorad, USA). The membranes were first subjected to overnight probing at $4^{\circ} \mathrm{C}$ with primary antibodies, followed by $60 \mathrm{~min}$ incubation with secondary antibodies conjugated to horseradish peroxidase at room temperature.

\section{Cell viability assay}

Cell viability was measured by the 3-(4,5-dimethylthiazol-2-yl)-2,5-diphenyltetrazolium bromide (MTT) assay. DBSCs were stimulated with a pulse (72h) of Res and Pol (Res 0.1-1.0 $\mu \mathrm{M}$ and Pol 0.1-1.0 $\mu \mathrm{M}$ ) during 7 days in osteogenic conditions. At the end of the culture $10 \mu \mathrm{l} /$ well of MTT $0.5 \mathrm{mg} / \mathrm{mL}$ were added, followed by $4-\mathrm{h}$ incubation at $37^{\circ} \mathrm{C}$ in a humidified 5\% $\mathrm{CO}_{2}$ chamber. The reaction was stopped by the addition of $100 \mu \mathrm{l}$ of $0.04 \mathrm{~N} \mathrm{HCl}$ in absolute isopropanol. The O.D. was read at $570 \mathrm{~nm}$ using an automatic plate reader (550 Microplate Reader Bio-Rad Laboratories Inc., CA, USA). The results were normalized to cells incubated under control conditions.

\section{Alkaline phosphatase (ALP)}

The expression of the biochemical marker for the osteoblastic activity ALP, was assessed in DBSCs, grown in osteogenic medium (a-MEM supplemented with $2 \%$ FBS, $10^{-8} \mathrm{M}$ dexamethasone, and $50 \mu \mathrm{g} / \mathrm{mL}$ ascorbic acid), with a commercial kit: Leukocyte Alkaline Phosphatase Kit (Sigma Aldrich). Cells were fixed with a solution provided from the kit according to manufacturer's instructions. After gently washed with deionized water, cells were stained in the dark with ALP solution (a mixture of FRV-Alkaline Solution, Naphthol AS-BI Alkaline Solution, $\mathrm{NaNO}_{2}$ ) for $15^{\prime}$, rinsed with water, air dried and then analyzed under the microscope. Osteoblasts positive for ALP show a purple color. ALP quantification was performed by ImageJ, analyzing the number of colored pixels corresponding to the positive stained cells.

\section{Alizarin red staining (ARS)}

The ability of differentiated DBSCs to generate calcium-rich deposits was detected by performing Alizarin Red Staining. The cell culture medium was removed before the staining procedure, the cells were rinsed gently with PBS and fixed with 10\% formalin at room temperature for 10 minutes. Fixative residues were removed with deionized water. $1 \%$ ARS solution was added and incubated at room temperature for 10 minutes. ARS solution was discarded, the wells were rinsed twice with deionized water and air dried. The monolayer appeared red stained. As previously described, the dye was extracted from the stained cell layer and assayed for quantification at $405 \mathrm{~nm}(41,42)$. The results were evaluated for statistical analysis.

\section{Statistical analyses}

Statistical analyses were performed by Student's t-test with the Statistical Package for the Social Sciences (spssx/pc) software (SPSS, Chicago, IL, USA). The results were considered statistically significant for $\mathrm{p}<0.05$.

\section{Results}

\section{DBSCs are Responsive to Res and Pol stimulation.}

Mesenchymal and stem features of DBSCs as well as commitment and differentiation toward osteoblastic lineage were established by flow cytometric analysis and in vitro osteoblast differentiation and mineralization. As already demonstrated (28), DBSCs from 15 donors confirmed $\geq 95 \%$ expression of CD73, CD90, CD105 and were negative for $\mathrm{CD} 45$, a common leukocyte antigen (data not shown). The cells, cultured under osteogenic conditions, differentiated into osteoblast-like cells, produced mineralized matrix nodules and expressed the typical osteoblastic markers Runx-2, Alkaline Phosphatase (ALP) and Collagen I (Coll I) (data not shown), thus reflecting the previously reported findings $(28,29)$. Res has been proposed as a potent stimulator of bone formation in vivo through its action in promoting osteoblast differentiation from MSCs $(15,16)$. Studies suggested that the natural precursor Pol, may have similar antioxidant properties, with the advantage of being more abundant and metabolically more stable (43-45). Res is known to regulate the Sirt-1 protein, by increasing its expression and activity (46, 47), thus before testing the role of these molecules on osteoblast differentiation of DBSCs, we tested the responsiveness of DBSCs to Res and Pol by investigating the expression of Sirt-1. The cells were seeded in osteogenic medium and after 4 days were stimulated with a pulse $(72 \mathrm{~h})$ of Res and Pol (Res 0.1-1.0 $\mu \mathrm{M}$ and Pol 0.1-1.0 $\mu \mathrm{M}$ ). At day 7 whole cell lysates were collected and analyzed by western blot. Sirt-1 was expressed in the control DBSCs differentiated for 7 days, but its expression was upregulated in the differentiated DBSCs treated with Res and Pol. The treatment with Res increased the expression of Sirt-1, without significant differences between the two doses $(0.1-1.0 \mu \mathrm{M}$; $55 \%-61 \%$ respectively), as did the treatment with $0.1 \mu \mathrm{M}$ Pol $(66 \%)$ and to a lesser extent with $1.0 \mu \mathrm{M}(17 \%)$ (Fig.1-A). These results demonstrated that DBSCs cultivated in osteogenic conditions are responsive to Res and Pol treatment by increasing the expression of Sirt-1. In parallel, cultures in same conditions were assayed for cell viability with MTT-test (3-(4,5-Dimethylthiazol-2-yl)-2,5-diphenyltetrazolium 


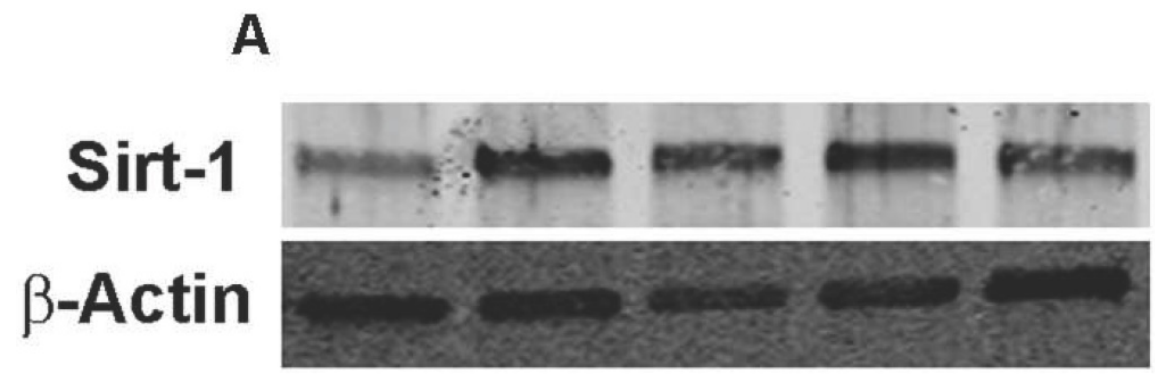

CTR RES 0.1 RES 1.0 POL 0.1 POL 1.0

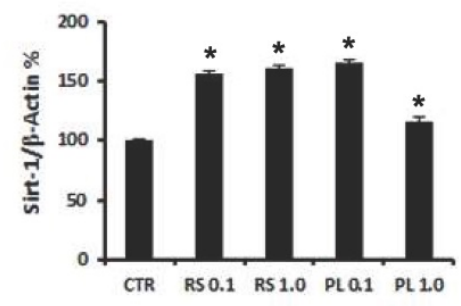

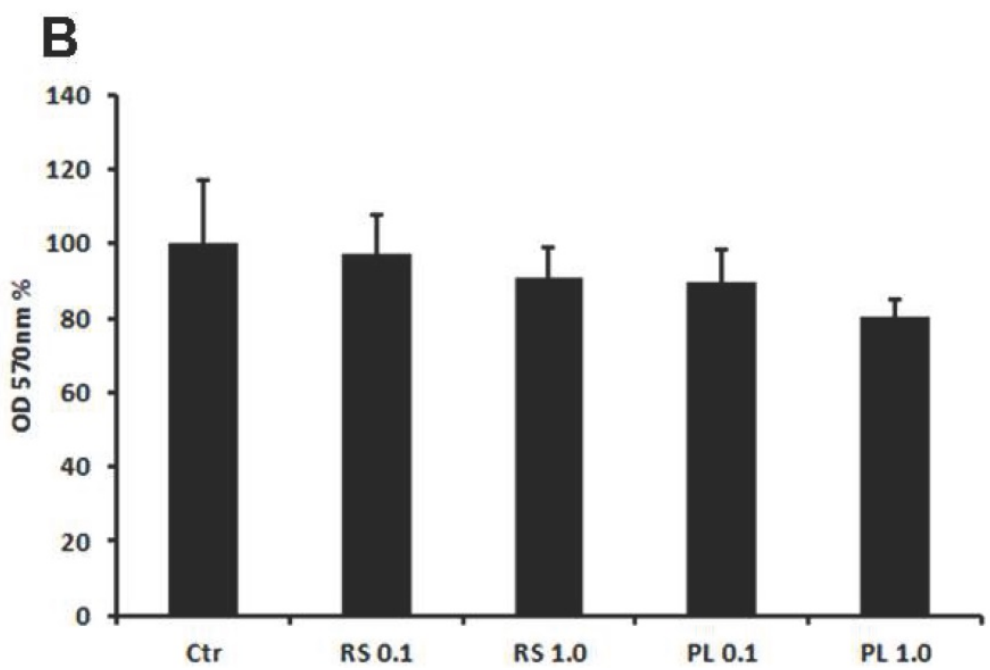

Fig.1. Responsiveness of DBSCs to Res and Pol stimulation and cell viability. (A) Immunoblots show the protein expression profile of Sirt-1, in DBSCs stimulated with a pulse (72h) of Res and Pol (Res 0.1-1.0 $\mu \mathrm{M}$ and Pol 0.1-1.0 $\mu \mathrm{M}$ ) during 7 days in osteogenic conditions and CTR. Each graph represents the mean OD + SE of 3 independent experiments performed in triplicate. $* P<0.01$ compared to T0. Student's $t$-test was used for single comparison. Representative immunoblotting were chosen for the figure. (B) MTT assay shows cell viability of DBSCs stimulated with a pulse (72h) of Res and Pol (Res 0.1-1.0 $\mu \mathrm{M}$ and Pol 0.1-1.0 $\mu$ M) during 7 days in osteogenic conditions and CTR. The graphs are representative of the mean values $\pm \mathrm{SE}$ of three independent experiments in which each treatment was performed in quadruplicate.

bromide). Fig.1-B showed that the treatment with Res (0.1-1.0 $\mu \mathrm{M})$ and Pol $(0.1-1.0 \mu \mathrm{M})$ did not affect significantly DBSCs growth or viability compared to control, although a slightly decrease of viability was observed in Pol $1.0 \mu \mathrm{M}$. By contrast, higher doses of both molecules $(10-100 \mu \mathrm{M})$ significantly decreased MTT values (data not shown).

\section{Res and Pol enhance osteogenic differentiation of DBSCs}

We investigated if the responsiveness of DBSCs to Res and Pol might influence their osteogenic differentiation. The cells were cultured in the same conditions described for the previous experiments and whole cell lysates were collected and subjected to western blot analysis. The expression of osteogenic differentiation markers ATF-4 and Osteopontin $(\mathrm{OPN})$ was investigated. Interestingly and consistently with the Sirt-1 modulation, Res treatment upregulated the expression of ATF-4 at both concentrations (0.1-1.0 $\mu \mathrm{M} ; 36 \%-46 \%$ respectively) while, Pol stimulation was efficacious to induce upregulation of ATF-4 at $0.1 \mu \mathrm{M}(23 \%)$ but not at 1.0 $\mu \mathrm{M}$ (Fig.2). Instead, Res treatment increased the expression of OPN at $1.0 \mu \mathrm{M}(63 \%)$ and interestingly, similar increase percentage was reached with lower concentration $(0.1 \mu \mathrm{M})$ of $\mathrm{Pol}(63 \%)$.

Therefore, we applied a histochemical assay to evaluate the expression of the typical osteoblast marker ALP. This test, reflecting the expression trend of OPN, showed that stimulation with Res $1.0 \mu \mathrm{M}$ and Pol $0.1 \mu \mathrm{M}$ increased ALP staining (Fig.3), thus indicating the ability of Res and Pol to enhance the differentiation of DBSCs in osteoblast-like cells and supporting the molecular profile results with phenotypical appearance. These results suggest a more responsiveness of the cells to Pol, indicating that Pol might be efficacious at a lower concentration than Res in inducing osteogenic differentiation of DBSCs.

\section{Res and Pol enhance mineral matrix nodules deposition of DBSCs}

To further explore the role of these molecules in osteoblast differentiation of DBSCs, we cultured the cells in mineralizing conditions with a pulse (72h) stimulation of Res and Pol. After 3 days in presence of mineralizing medium the cells were stimulated with Res 0.1-1.0 $\mu \mathrm{M}$ and Pol 0.1-1.0 $\mu \mathrm{M}$ for $72 \mathrm{~h}$ and kept in 
culture until bone matrix deposition. Using histochemical assays, we evaluated with Alizarin Red Staining (ARS) how Res and Pol stimulation influenced the mineralization capacity of DBSCs. The mineral matrix nodules deposition capacity was enhanced in cells treated with $0.1 \mu \mathrm{M}$ Pol in comparison to the control (116\%), while $1.0 \mu \mathrm{M}$ dose of Pol didn't change the ability of the cells to mineralize. The treatment with Res increased the mineral matrix deposition of DBSCs with a mild but not significant difference between the two doses (0.1-1.0 $\mu \mathrm{M}$; 86-65\%) (Fig.4). Interestingly, although here the lower dose of Res $(0.1 \mu \mathrm{M})$, was sufficient to rise mineral deposition, ARS quantification revealed that mineral matrix deposition was higher (30-52\%) in cells cultured with Pol $0.1 \mu \mathrm{M}$ if compared to cells treated with Res 0.1-1.0 $\mu \mathrm{M}$.
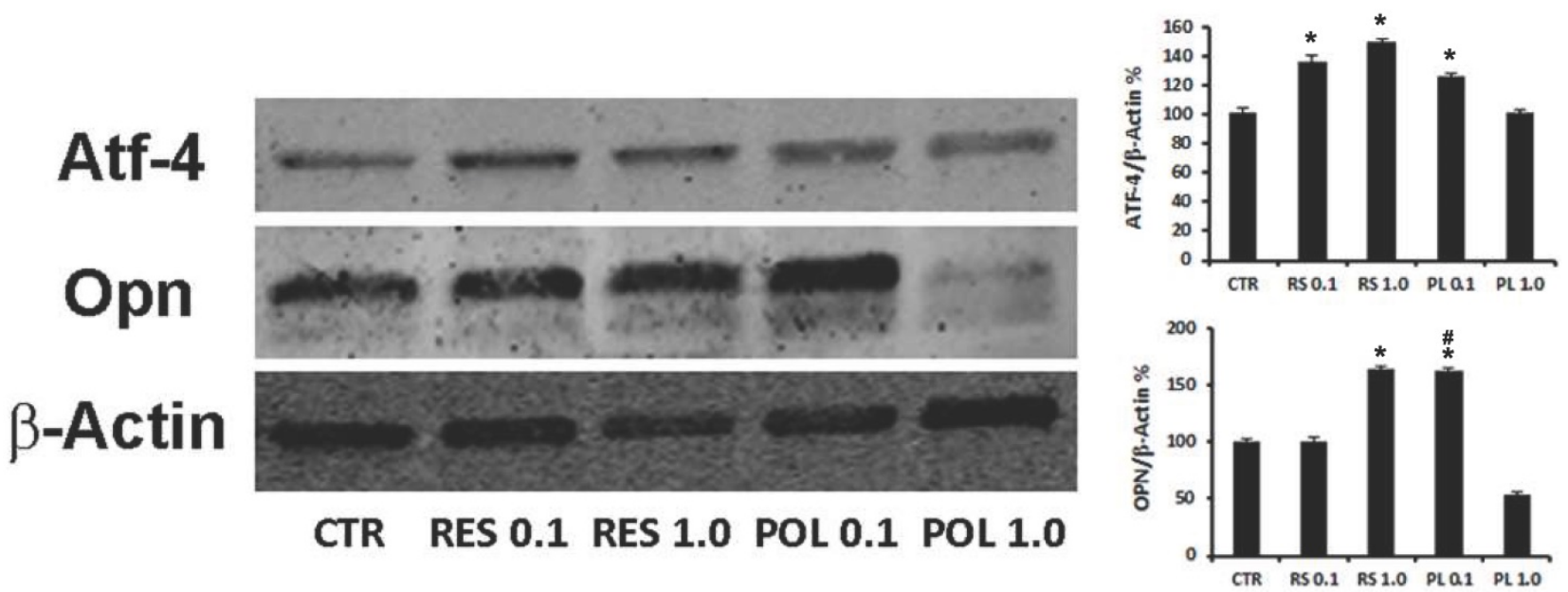

Fig. 2. Effect of Res and Pol on the expression of osteogenic markers ATF-4 and OPN. Immunoblots show the protein expression profile of two markers of osteogenic differentiation, ATF-4 and OPN, in DBSCs stimulated with a pulse (72h) of Res and Pol (Res 0.1-1.0 $\mu \mathrm{M}$ and Pol 0.1-1.0 $\mu \mathrm{M}$ ) during 7 days in osteogenic conditions and CTR. Each graph represents the mean OD \pm SE of 3 independent experiments performed in triplicate. $* P<0.01$ compared to T0. \#P $<0.01$ compared to Res $0.1 \mu M$. Student's $t$-test was used for single comparison. Representative immunoblotting were chosen for the figure.

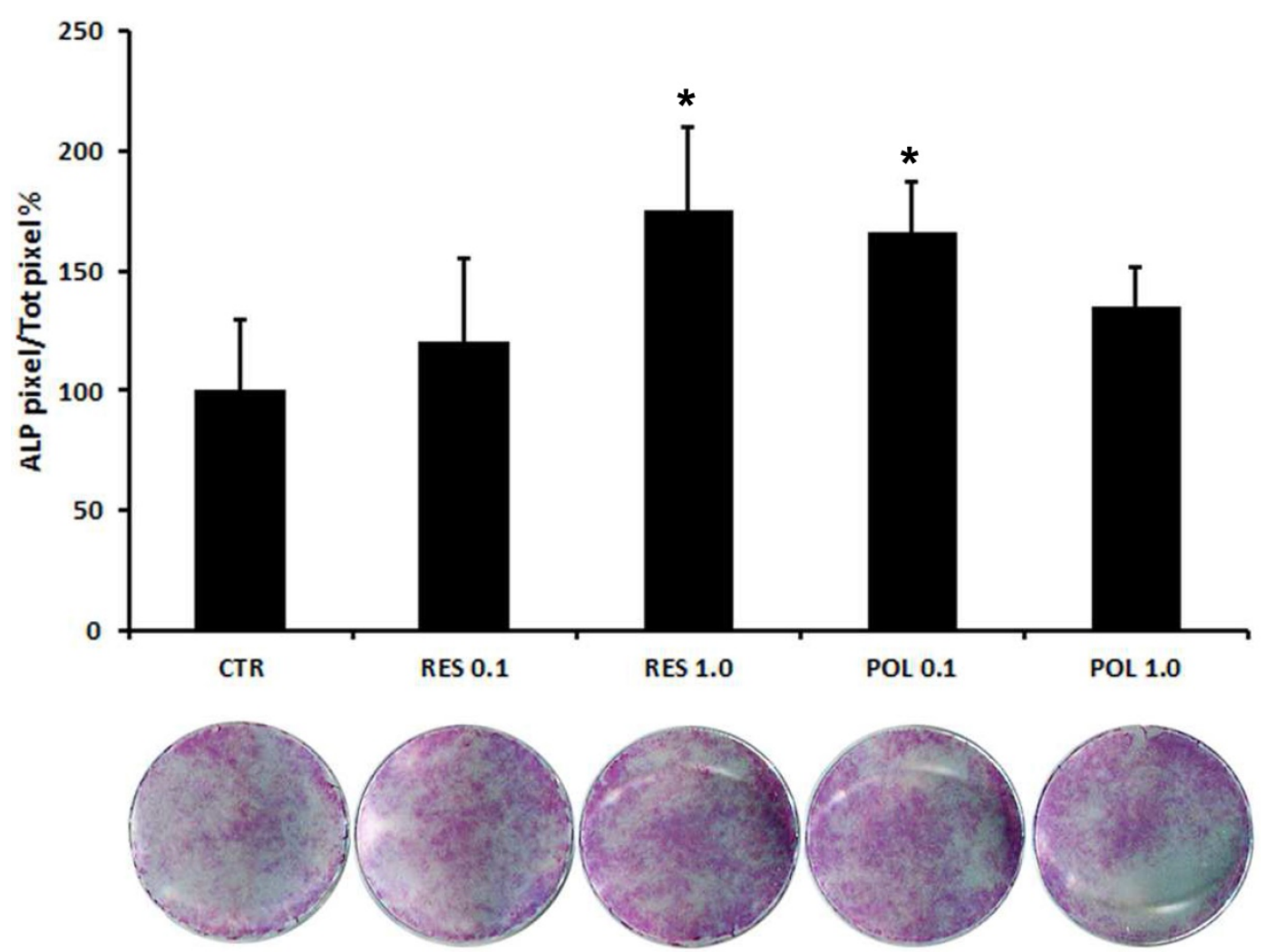

Fig. 3. Expression of osteogenic marker ALP in response to Res and Pol stimulation. ALP histochemical assay (purple staining) performed on DBSCs stimulated with

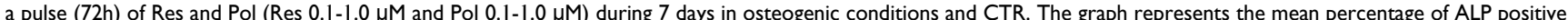
area/well \pm SE of 3 independent experiments performed in quadruplicate. $(* P<001)$ compared to CTR. Student's $t$-test was used for single comparisons. The wells of a representative experiment were chosen for the figure. 


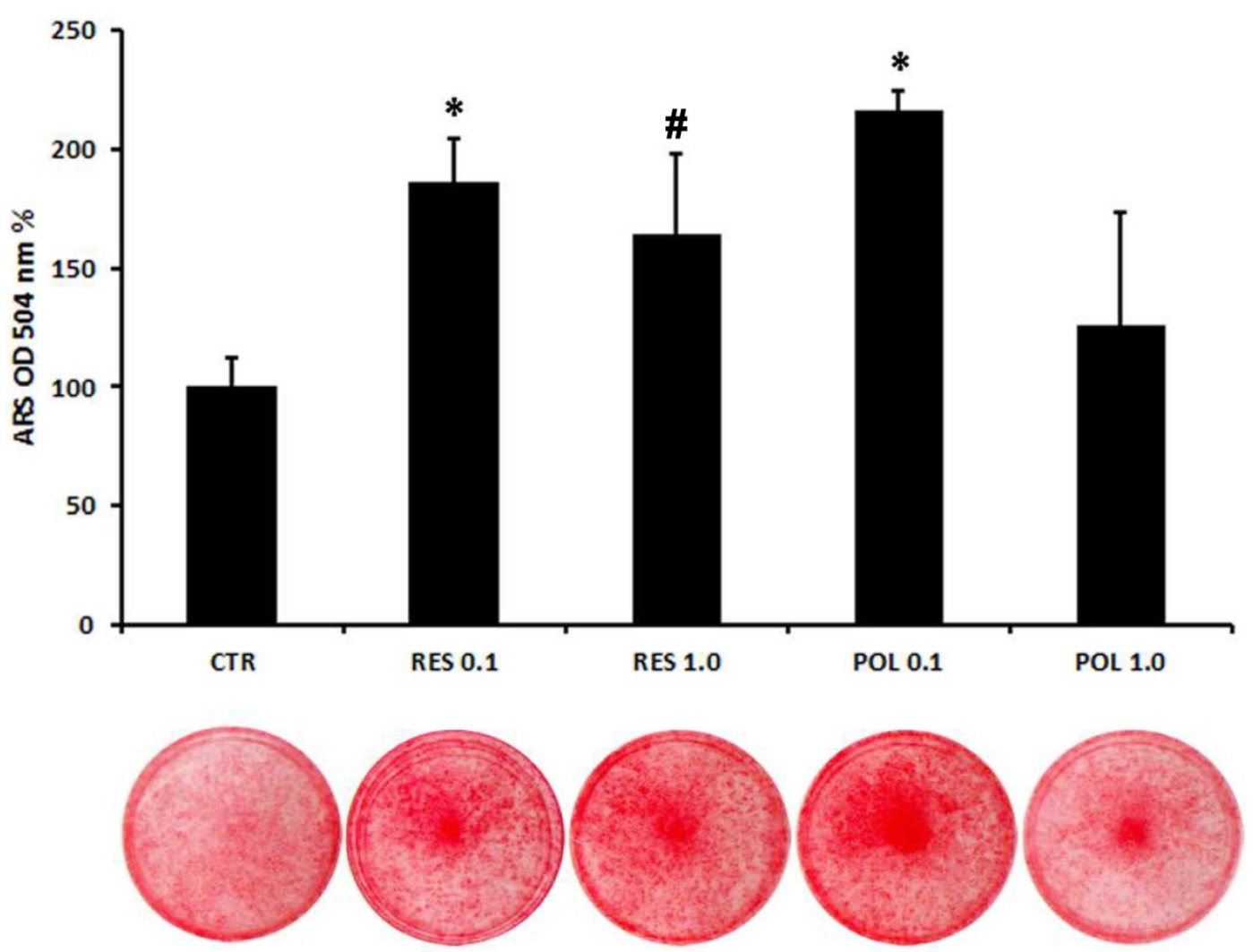

Fig. 4. Quantification of mineral matrix deposition following Res and Pol stimulation. Mineral matrix deposition was assayed after 21 days in osteogenic conditions by ARS (red staining). The graph shows the OD quantification of extracted dye from stained cell layers as mean percentage \pm SE of 3 independent experiments performed in quadruplicate. $* P<0,001 ; \# P<0.05$ compared to CTR. Student's $t$-test was used for single comparisons. The wells of a representative experiment were chosen for the figure.

\section{Discussion}

The results of the present study demonstrated for the first time that Pol, the natural glycosilated precursor of Res, is able to stimulate the osteogenic differentiation of human MSCs from dental bud. As we previously demonstrated $(28,29)$ DBSCs expressed $\geq 95 \%$ of common mesenchymal stem markers (CD73, CD90, CD105), under osteogenic conditions expressed the typical osteoblastic markers and produced mineralized matrix nodules, representing an optimal model of cell differentiation toward osteogenic lineage. Recently we also showed that natural molecules, as vitamin $\mathrm{D}$, oxytocin, further increased the osteogenic potential of mesenchymal and osteoblast-like cells (48-52). First, here we showed that DBSCs were responsive to Res and Pol, since the treatment with both molecules was able to increase the expression of Sirt-1. Res is known to modulate Sirt-1 expression and activity in different cell types $(15,46,47)$ including MSCs from human bone marrow $(53,54)$ and dental pulp (55); Pol was reported more recently to increase Sirt-1 levels in some cell types (56-58) but no data were present about MSCs. Our results are then consistent with the literature data, but in addition provide the first evidence that human MSCs respond to Pol stimulation by increasing Sirt-1 expression, likewise to Res. Subsequently we have demonstrated that DBSCs cultured in osteogenic conditions responded to Res and Pol stimulation by increasing the expression of the typical osteoblast marker Atf-4. Atf-4 is a transcription factor required for osteoblast differentiation that in turn regulates the expression of other osteoblastic proteins such as the extracellular matrix components (59-61). Furthermore, our experiments demonstrated that the expression of OPN, a typical bone matrix protein, was upregulated by the treatment with Res and Pol. These findings showed that Res and Pol stimulation increased Sirt-1 expression in DBSCs driving these cells, through ATF-4, toward osteoblast maturation, although the intracellular signaling mechanism needs deeper investigations. Interestingly is reported that Sirt-1 promotes osteogenic differentiation and is upregulated during the osteogenic process (62-64), thus supporting our finding. The present study further provided demonstrations that this molecular pattern was actually accompanied by enzymatic expression evidences. Indeed DBSCs treated with Res and Pol showed increased expression of ALP, the enzyme promoting bone matrix mineralization (65) and consistent with this, the treated cells showed higher amount of mineral matrix deposition. It is known that Res stimulates in vivo bone formation (17, 18) promoting in vitro the differentiation of osteoblasts 
and precursors $(9,13-16)$. To establish efficacious dosage ranges of administration for human trials has been difficult $(19,20)$ because some studies have demonstrated that intestinal absorption of Res is high, but its bioavailability is low due to its rapid metabolism $(66,67)$. Whereas the presence of a glycosilic group in the Pol molecule allows to resist oxidation, prolongs its half-life and increases its solubility (68), furthermore Pol amount results to be more abundant then Res in the plants (22-24). The outcome of this work is that any data were present in the literature regarding the effect of Pol on osteoblast differentiation, while we showed that the glucoside shared similar effects with Res, enhancing osteogenic differentiation of DBSCs and increasing mineral matrix deposition. Similarly, Pol shares with Res antioxidant $(25,69-71)$ and anti-inflammatory $(39,72$, 73) features, although appearing even more efficacious in modulating oxidative stress $(21,70)$. Intriguingly we showed that lower dose of Pol (0.1 $\mu \mathrm{M})$ consistently stimulates the osteogenic differentiation of DBSCs, up-regulating the expression of typical osteoblast markers (ATF-4, OCN, ALP) and increasing mineral matrix deposition, while same dose of Res is not always efficacious and the higher dose $(1.0 \mu \mathrm{M})$ is somehow necessary to raise the expression at least of OCN and ALP. Notably, both concentrations $(0.1-1.0 \mu \mathrm{M})$ of Res and Pol used in this work, did not affect significantly DBSCs growth or viability (Fig.1-B), although a slightly decrease of viability is observed in Pol $1.0 \mu \mathrm{M}$, as did higher concentrations of both molecules (10-100 $\mu \mathrm{M})$ (data not shown). Importantly, dose depending effects have been reported in the literature for Res (74-78), likewise recently emerged for Pol $(26,27)$. In the light of these evidences it is possible to hypothesize that concentrations $\geq 1.0 \mu \mathrm{M}$ might be ineffective, or even inhibitory on osteogenic differentiation of MSCs from dental bud. Actually Pol $0.1 \mu \mathrm{M}$ significantly increased all the tested markers, while Pol $1.0 \mu \mathrm{M}$ appeared to be mostly ineffective on osteogenic differentiation, probably due to a slight inhibition on cell viability. Res $0.1 \mu \mathrm{M}$, in some cases (OPN expression, ALP expression) seemed to be inefficacious (Fig.2-3). Such markers indeed, increased with the higher $(1.0 \mu \mathrm{M})$ concentration of Res. In the perspective of using molecules, although of natural origin, for therapeutic purposes, it is preferable efficacy at low doses, rather than high, in order to avoid the drawbacks due to high doses. In the light of this, Pol might be considered highly effective because elicited positive significant effects on all osteogenic markers tested, at $0.1 \mu \mathrm{M}$ concentration. While, Res failed to elicit significant increase of OPN and ALP at $0.1 \mu \mathrm{M}$. These evidences, the metabolic stability and antioxidant features of Pol, encourage to further explore the effects of this molecule on bone metabolism and on its associated mechanisms of action. Interestingly, Pol elicited intriguing effects on bone-related cells, since protects BMSCs survival against oxidative stress (26) and inhibits the growth of osteosarcoma cells (27) at different concentrations. Thus, in conclusion, it is conceivable a role of Pol in ameliorating bone health as elective molecule for non-pharmacological therapies, such as those based on the consumption of functional food.

\section{Abbreviations}

MSCs: Mesenchymal Stem Cells; DBSCs: Dental Bud Stem Cells; Res: Resveratrol; Pol: Polydatin; ALP: Alkaline Phosphatase; ARS: Alizarin Red Staining; Coll I: Collagen I; MTT: 3-(4,5-Dimethylthiazol-2-yl)2,5-diphenyltetrazolium bromide; OPN: Osteopontin; BMSCs: Bone Marrow Stem/Stromal Cells.

\section{Acknowledgements}

\section{Funding}

The author A.D. is funded by Fondo di Sviluppo e Coesione 2007-2013, APQ Ricerca Regione Puglia "Programma regionale a sostegno della specializzazione intelligente e della sostenibilità sociale ed ambientale-FutureInResearch". This work was partially awarded from Ministero dell'Istruzione, dell'Università e della Ricerca-PRIN 20098KM9RN (Progetto di Ricerca d'Interesse Nazionale-Grant 2009).

\section{Authorship}

A.D. and G.M. conceived and designed the research; A.D. and F.P. performed the research; A.D., F.P. and G.M. analyzed the data; S.D., G.R., A.B., C.P., T.T, M.G., L.L. contributed to reagents, materials and analysis tools; A.D., F.P., G.M., G.R. wrote the paper. All authors read and approved the final manuscript.

\section{Disclaimer}

The present work was extracted from the Patent n.16999PTIT entitled "Composizioni comprendenti o costituite da Polidatina per uso nel trattamento delle patologie ossee", deposited with application number 102017000079581.

\section{Competing Interests}

The authors have declared that no competing interest exists.

\section{References}

1. Bernabei R, Martone AM, Ortolani E, Landi F, Marzetti E. Screening, diagnosis and treatment of osteoporosis: a brief review. Clinical cases in mineral and bone metabolism : the official journal of the Italian Society of Osteoporosis, Mineral Metabolism, and Skeletal Diseases. 2014;11(3):201-7. 
2. Gunn CA, Weber JL, McGill AT, Kruger MC. Increased intake of selected vegetables, herbs and fruit may reduce bone turnover in post-menopausal women. Nutrients. 2015;7(4):2499-517.

3. Benetou V, Orfanos P, Feskanich D, Michaelsson K, Pettersson-Kymmer U, Eriksson S, et al. Fruit and Vegetable Intake and Hip Fracture Incidence in Older Men and Women: The CHANCES Project. Journal of bone and mineral research : the official journal of the American Society for Bone and Mineral Research. 2016.

4. Dodington DW, Fritz PC, Sullivan PJ, Ward WE. Higher Intakes of Fruits and Vegetables, beta-Carotene, Vitamin C, alpha-Tocopherol, EPA, and DHA Are Positively Associated with Periodontal Healing after Nonsurgical Periodontal Therapy in Nonsmokers but Not in Smokers. J Nutr. 2015;145(11):2512-9.

5. Conti V, Izzo V, Corbi G, Russomanno G, Manzo V, De Lise F, et al. Antioxidant Supplementation in the Treatment of Aging-Associated Diseases. Frontiers in pharmacology. 2016;7:24.

6. Gambini J, Ingles M, Olaso G, Lopez-Grueso R, Bonet-Costa V, Gimeno-Mallench L, et al. Properties of Resveratrol: In Vitro and In Vivo Studies about Metabolism, Bioavailability, and Biological Effects in Animal Models and Humans. Oxidative medicine and cellular longevity. 2015;2015:837042.

7. Porro C, Cianciulli A, Calvello R, Panaro MA. Reviewing the Role of Resveratrol as a Natural Modulator of Microglial Activities. Current pharmaceutical design. 2015;21(36):5277-91.

8. Juhasz B, Varga B, Gesztelyi R, Kemeny-Beke A, Zsuga J, Tosaki A. Resveratrol: a multifunctional cytoprotective molecule. Current pharmaceutical biotechnology. 2010;11(8):810-8.

9. Mizutani K, Ikeda K, Kawai Y, Yamori Y. Resveratrol stimulates the proliferation and differentiation of osteoblastic MC3T3-E1 cells. Biochemical and biophysical research communications. 1998;253(3):859-63.

10. He N, Zhu X, He W, Zhao S, Zhao W, Zhu C. Resveratrol inhibits the hydrogen dioxide-induced apoptosis via Sirt 1 activation in osteoblast cells. Bioscience, biotechnology, and biochemistry. 2015;79(11):1779-86.

11. Kuroyanagi G, Tokuda H, Yamamoto N, Matsushima-Nishiwaki R, Mizutani $\mathrm{J}$, Kozawa $\mathrm{O}$, et al. Resveratrol amplifies BMP-4-stimulated osteoprotegerin synthesis via p38 MAP kinase in osteoblasts. Molecular medicine reports. 2015;12(3):3849-54

12. Rucinski M, Ziolkowska A, Hochol A, Pucher A, Macchi C, Belloni AS, et al. Estradiol and resveratrol stimulating effect on osteocalcin, but not osteonectin and collagen-1alpha gene expression in primary culture of rat calvarial osteoblast-like cells. International journal of molecular medicine. 2006;18(4):565-70.

13. Zhou H, Shang L, Li X, Zhang X, Gao G, Guo C, et al. Resveratrol augments the canonical Wnt signaling pathway in promoting osteoblastic differentiation of multipotent mesenchymal cells. Experimental cell research. 2009.315(17):2953-62

14. Lee YM, Shin SI, Shin KS, Lee YR, Park BH, Kim EC. The role of sirtuin 1 in osteoblastic differentiation in human periodontal ligament cells. Journal of periodontal research. 2011;46(6):712-21.

15. Shakibaei M, Shayan P, Busch F, Aldinger C, Buhrmann C, Lueders C, et al. Resveratrol mediated modulation of Sirt-1/Runx2 promotes osteogenic differentiation of mesenchymal stem cells: potential role of Runx2 deacetylation. PloS one. 2012;7(4):e35712.

16. Ornstrup MJ, Harslof T, Sorensen L, Stenkjaer L, Langdahl BL, Pedersen SB. Resveratrol Increases Osteoblast Differentiation In Vitro Independently of Inflammation. Calcified tissue international. 2016.

17. Tou JC. Resveratrol supplementation affects bone acquisition and osteoporosis: Pre-clinical evidence toward translational diet therapy. Biochimica et biophysica acta. 2015;1852(6):1186-94.

18. Shen $\mathrm{CL}$, von Bergen $\mathrm{V}$, Chyu $\mathrm{MC}$, Jenkins $\mathrm{MR}, \mathrm{Mo} \mathrm{H}$, Chen $\mathrm{CH}$, et al. Fruits and dietary phytochemicals in bone protection. Nutrition research (New York, NY). 2012;32(12):897-910.

19. Amri A, Chaumeil JC, Sfar $S$, Charrueau $C$ Administration of resveratrol: What formulation solutions to bioavailability limitations? Journal of controlled release : official journal of the Controlled Release Society. 2012;158(2):182-93.

20. Novelle MG, Wahl D, Dieguez C, Bernier M, de Cabo R. Resveratrol supplementation: Where are we now and where should we go? Ageing research reviews. 2015;21:1-15.

21. Wang HL, Gao JP, Han YL, Xu X, Wu R, Gao Y, et al. Comparative studies of polydatin and resveratrol on mutual transformation and antioxidative effect in vivo. Phytomedicine : international journal of phytotherapy and phytopharmacology. 2015;22(5):553-9.

22. Chen M, Li D, Gao Z, Zhang C. Enzymatic transformation of polydatin to resveratrol by piceid-beta-D-glucosidase from Aspergillus oryzae. Bioprocess and biosystems engineering. 2014;37(7):1411-6.

23. Xie L, Bolling BW. Characterisation of stilbenes in California almonds (Prunus dulcis) by UHPLC-MS. Food chemistry. 2014;148:300-6.

24. Peng $\mathrm{XL}, \mathrm{Xu} \mathrm{J}$, Sun $\mathrm{XF}$, Ying CJ, Hao LP. Analysis of trans-resveratrol and trans-piceid in vegetable foods using high-performance liquid chromatography. International journal of food sciences and nutrition. 2015;66(7):729-35.

25. De Maria S, Scognamiglio I, Lombardi A, Amodio N, Caraglia M, Carteni M, et al. Polydatin, a natural precursor of resveratrol, induces cell cycle arrest and differentiation of human colorectal Caco-2 cell. Journal of translational medicine. 2013;11:264
26. Chen M, Hou Y, Lin D. Polydatin Protects Bone Marrow Stem Cells against Oxidative Injury: Involvement of Nrf 2/ARE Pathways. Stem cells international. 2016;2016:9394150.

27. Xu G, Kuang G, Jiang W, Jiang R, Jiang D. Polydatin promotes apoptosis through upregulation the ratio of $\mathrm{Bax} / \mathrm{Bcl}-2$ and inhibits proliferation by attenuating the beta-catenin signaling in human osteosarcoma cells. American journal of translational research. 2016;8(2):922-31.

28. Di Benedetto A, Brunetti G, Posa F, Ballini A, Grassi FR, Colaianni G, et al. Osteogenic differentiation of mesenchymal stem cells from dental bud: Role of integrins and cadherins. Stem cell research. 2015;15(3):618-28.

29. Mori G, Ballini A, Carbone C, Oranger A, Brunetti G, Di Benedetto A, et al. Osteogenic differentiation of dental follicle stem cells. International journal of medical sciences. 2012;9(6):480-7.

30. Mori G, Brunetti G, Oranger A, Carbone C, Ballini A, Lo Muzio L, et al. Dental pulp stem cells: osteogenic differentiation and gene expression. Annals of the New York Academy of Sciences. 2011;1237:47-52.

31. Giorgini E, Conti C, Ferraris P, Sabbatini S, Tosi G, Centonze M, et al. FT-IR microscopic analysis on human dental pulp stem cells. Vibrational Spectroscopy. 2011;57(1):30-4

32. Mori G, Centonze M, Brunetti G, Ballini A, Oranger A, Mori C, et al. Osteogenic properties of human dental pulp stem cells. Journal of biological regulators and homeostatic agents. 2010;24(2):167-75

33. Graziano A, d'Aquino R, Laino G, Papaccio G. Dental pulp stem cells: a promising tool for bone regeneration. Stem cell reviews. 2008;4(1):21-6.

34. d'Aquino R, De Rosa A, Laino G, Caruso F, Guida L, Rullo R, et al. Human dental pulp stem cells: from biology to clinical applications. Journal of experimental zoology Part B, Molecular and developmental evolution. 2009;312b(5):408-15.

35. Mangano C, De Rosa A, Desiderio V, d'Aquino R, Piattelli A, De Francesco F, et al. The osteoblastic differentiation of dental pulp stem cells and bone formation on different titanium surface textures. Biomaterials. 2010;31(13):3543-51.

36. Brunetti G, Oranger A, Carbone C, Mori G, Sardone FR, Mori C, et al. Osteoblasts display different responsiveness to TRAIL-induced apoptosis during their differentiation process. Cell Biochem Biophys. 2013;67(3):1127-36.

37. Mori G, Brunetti G, Ballini A, Di Benedetto A, Tarantino U, Colucci S, et al. Biological characteristics of dental stem cells for tissue engineering. Key Engineering Materials; 2013: Trans Tech Publ.

38. Brunetti G, Di Benedetto A, Posa F, Colaianni G, Faienza MF, Ballini A, et al. High expression of TRAIL by osteoblastic differentiated dental pulp stem cells affects myeloma cell viability. Oncology reports. 2018;39(4):2031-9.

39. Ravagnan G, De Filippis A, Carteni M, De Maria S, Cozza V, Petrazzuolo M, et al. Polydatin, a natural precursor of resveratrol, induces beta-defensin production and reduces inflammatory response. Inflammation. 2013;36(1):26-34

40. Di Benedetto A, Carbone C, Mori G. Dental pulp stem cells isolation and osteogenic differentiation: a good promise for tissue engineering. Methods in molecular biology. 2014;1210:117-30.

41. Di Benedetto A, Posa F. NURR1 Downregulation Favors Osteoblastic Differentiation of MSCs. 2017;2017:7617048.

42. Gregory CA, Gunn WG, Peister A, Prockop DJ. An Alizarin red-based assay of mineralization by adherent cells in culture: comparison with cetylpyridinium chloride extraction. Analytical biochemistry. 2004;329(1):77-84.

43. Mark L, Nikfardjam MS, Avar P, Ohmacht R. A validated HPLC method for the quantitative analysis of trans-resveratrol and trans-piceid in Hungarian wines. Journal of chromatographic science. 2005;43(9):445-9.

44. Wang H, Liu L, Guo YX, Dong YS, Zhang DJ, Xiu ZL. Biotransformation of piceid in Polygonum cuspidatum to resveratrol by Aspergillus oryzae. Applied microbiology and biotechnology. 2007;75(4):763-8.

45. Zhou L, Li S, Zhang T, Mu W, Jiang B. Properties of a novel polydatin-beta-d-glucosidase from Aspergillus niger SK34.002 and its application in enzymatic preparation of resveratrol. Journal of the science of food and agriculture. 2016;96(7):2588-95

46. Bagul PK, Dinda AK, Banerjee SK. Effect of resveratrol on sirtuins expression and cardiac complications in diabetes. Biochemical and biophysical research communications. 2015;468(1-2):221-7.

47. Howitz KT, Bitterman KJ, Cohen HY, Lamming DW, Lavu S, Wood JG, et al. Small molecule activators of sirtuins extend Saccharomyces cerevisiae lifespan. Nature. 2003:425(6954):191-6.

48. Posa F, Di Benedetto A, Colaianni G. Vitamin D Effects on Osteoblastic Differentiation of Mesenchymal Stem Cells from Dental Tissues. 2016;2016:9150819.

49. Di Benedetto A, Sun L, Zambonin CG, Tamma R, Nico B, Calvano CD, et al. Osteoblast regulation via ligand-activated nuclear trafficking of the oxytocin receptor. Proceedings of the National Academy of Sciences of the United States of America. 2014;111(46):16502-7.

50. Sun L, Tamma R, Yuen T, Colaianni G, Ji Y, Cuscito C, et al. Functions of vasopressin and oxytocin in bone mass regulation. Proceedings of the National Academy of Sciences of the United States of America. 2016;113(1):164-9.

51. Colaianni G, Tamma R, Di Benedetto A, Yuen T, Sun L, Zaidi M, et al. The oxytocin-bone axis. Journal of neuroendocrinology. 2014;26(2):53-7.

52. Posa F, Di Benedetto A, Cavalcanti-Adam EA, Colaianni G, Porro C, Trotta T, et al. Vitamin D Promotes MSC Osteogenic Differentiation Stimulating Cell 
Adhesion and \&\#x03B1;V\&\#x03B2;3 Expression. Stem cells international. 2018;2018:9.

53. Yoon DS, Choi Y, Jang Y, Lee M, Choi WJ, Kim SH, et al. SIRT1 directly regulates SOX2 to maintain self-renewal and multipotency in bone marrow-derived mesenchymal stem cells. Stem cells (Dayton, Ohio). 2014;32(12):3219-31.

54. Yuan HF, Zhai C, Yan XL, Zhao DD, Wang JX, Zeng Q, et al. SIRT1 is required for long-term growth of human mesenchymal stem cells. Journal of molecular medicine (Berlin, Germany). 2012;90(4):389-400.

55. Feng G, Zheng K, Song D, Xu K, Huang D, Zhang Y, et al. SIRT1 was involved in TNF-alpha-promoted osteogenic differentiation of human DPSCs through Wnt/beta-catenin signal. In vitro cellular \& developmental biology Animal. 2016.

56. Huang K, Chen C, Hao J, Huang J, Wang S, Liu P, et al. Polydatin promotes Nrf2-ARE anti-oxidative pathway through activating Sirt1 to resist AGEs-induced upregulation of fibronetin and transforming growth factor-beta1 in rat glomerular messangial cells. Molecular and cellular endocrinology. 2015;399:178-89.

57. Chen L, Lan Z. Polydatin attenuates potassium oxonate-induced hyperuricemia and kidney inflammation by inhibiting NF-kappaB/NLRP3 inflammasome activation via the AMPK/SIRT1 pathway. Food \& function. 2017;8(5):1785-92.

58. Li P, Liu Y, Burns N, Zhao KS, Song R. SIRT1 is required for mitochondrial biogenesis reprogramming in hypoxic human pulmonary arteriolar smooth muscle cells. International journal of molecular medicine. 2017;39(5):1127-36.

59. Yang X, Matsuda K, Bialek P, Jacquot S, Masuoka HC, Schinke T, et al. ATF4 is a substrate of RSK2 and an essential regulator of osteoblast biology; implication for Coffin-Lowry Syndrome. Cell. 2004;117(3):387-98.

60. Xiao G, Jiang D, Ge C, Zhao Z, Lai $Y$, Boules $\mathrm{H}$, et al. Cooperative interactions between activating transcription factor 4 and Runx2/Cbfa1 stimulate osteoblast-specific osteocalcin gene expression. The Journal of biological chemistry. 2005;280(35):30689-96

61. Huang W, Yang S, Shao J, Li YP. Signaling and transcriptional regulation in osteoblast commitment and differentiation. Frontiers in bioscience : a journal and virtual library. 2007;12:3068-92.

62. Li M, Yan J, Chen X, Tam W, Zhou L, Liu T, et al. Spontaneous up-regulation of SIRT1 during osteogenesis contributes to stem cells' resistance to oxidative stress. Journal of cellular biochemistry. 2018.

63. Zainabadi K, Liu CJ, Guarente L. SIRT1 is a positive regulator of the master osteoblast transcription factor, RUNX2. PloS one. 2017;12(5):e0178520.

64. Qu B, Ma Y, Yan M, Gong K, Liang F, Deng S, et al. Sirtuin1 promotes osteogenic differentiation through downregulation of peroxisome proliferator-activated receptor gamma in MC3T3-E1 cells. Biochemical and biophysical research communications. 2016;478(1):439-45.

65. Rodan GA, Noda M. Gene expression in osteoblastic cells. Critical reviews in eukaryotic gene expression. 1991;1(2):85-98.

66. Almeida L, Vaz-da-Silva M, Falcao A, Soares E, Costa R, Loureiro AI, et al. Pharmacokinetic and safety profile of trans-resveratrol in a rising multiple-dose study in healthy volunteers. Molecular nutrition \& food research. 2009;53 Suppl 1:S7-15.

67. Cottart CH, Nivet-Antoine V, Laguillier-Morizot C, Beaudeux JL. Resveratrol bioavailability and toxicity in humans. Molecular nutrition \& food research. 2010;54(1):7-16.

68. Regev-Shoshani G, Shoseyov O, Bilkis I, Kerem Z. Glycosylation of resveratrol protects it from enzymic oxidation. The Biochemical journal. 2003;374(Pt 1):157-63.

69. Ince S, Arslan Acaroz D, Neuwirth O, Demirel HH, Denk B, Kucukkurt I, et al. Protective effect of polydatin, a natural precursor of resveratrol, against cisplatin-induced toxicity in rats. Food and chemical toxicology : an international journal published for the British Industrial Biological Research Association. 2014;72:147-53.

70. Fabris S, Momo F, Ravagnan G, Stevanato R. Antioxidant properties of resveratrol and piceid on lipid peroxidation in micelles and monolamellar liposomes. Biophysical chemistry. 2008;135(1-3):76-83.

71. Pace MC, Passavanti MB, Aurilio C, Sansone P, Aurilio R, S DEM, et al. Polydatin administration improves serum biochemical parameters and oxidative stress markers during chronic alcoholism: a pilot study. In vivo (Athens, Greece). 2015;29(3):405-8

72. Zhang $\mathrm{L}$, Li Y, Gu Z, Wang Y, Shi M, Ji Y, et al. Resveratrol inhibits enterovirus 71 replication and pro-inflammatory cytokine secretion in rhabdosarcoma cells through blocking IKKs/NF-kappaB signaling pathway. PloS one. 2015;10(2): $\mathrm{e} 0116879$

73. Lanzilli G, Cottarelli A, Nicotera G, Guida S, Ravagnan G, Fuggetta MP. Anti-inflammatory effect of resveratrol and polydatin by in vitro IL-17 modulation. Inflammation. 2012;35(1):240-8.

74. Li Q, Huyan T, Ye LJ, Li J, Shi JL, Huang QS. Concentration-dependent biphasic effects of resveratrol on human natural killer cells in vitro. Journal of agricultural and food chemistry. 2014;62(45):10928-35.

75. Erdman CP, Dosier CR, Olivares-Navarrete R, Baile C, Guldberg RE, Schwartz $Z$, et al. Effects of resveratrol on enrichment of adipose-derived stem cells and their differentiation to osteoblasts in two-and three-dimensional cultures. Journal of tissue engineering and regenerative medicine. 2012;6 Suppl $3 \cdot s 34-46$.
76. Su D, Cheng Y, Liu M, Liu D, Cui H, Zhang B, et al. Comparision of piceid and resveratrol in antioxidation and antiproliferation activities in vitro. PloS one. 2013;8(1):e54505.

77. Gueguen N, Desquiret-Dumas V, Leman G, Chupin S, Baron S, Nivet-Antoine $\mathrm{V}$, et al. Resveratrol Directly Binds to Mitochondrial Complex I and Increases Oxidative Stress in Brain Mitochondria of Aged Mice. PloS one. 2015;10(12):e0144290.

78. Falchetti R, Fuggetta MP, Lanzilli G, Tricarico M, Ravagnan G. Effects of resveratrol on human immune cell function. Life sciences. 2001;70(1):81-96. 